\title{
Villoglandular papillary adenocarcinoma of the uterine cervix: A report of 4 cases and a review of the literature
}

\author{
QIANG-YONG ZHOU, HAI-YAN CHEN, SI-MENG YANG, YUE-HUA LI and XUE-QING WU \\ Department of Obstetrics and Gynecology, The First Affiliated Hospital of Wenzhou Medical University, \\ Wenzhou, Zhejiang 325000, P.R. China
}

Received September 19, 2014; Accepted May 15, 2015

DOI: 10.3892/ol.2015.3944

\begin{abstract}
To investigate the clinicopathological features, management and prognosis of villoglandular papillary adenocarcinoma (VGPA) of the uterine cervix, the current study presents 4 cases of VGPA of the uterine cervix. The median age of the patients was 55 years (range, 47-70 years), with all 4 patients presenting with stage Ib disease. Human papillomavirus (HPV) infection was detected in 3 patients; this was mainly HPV-16. No history of oral contraceptive use was found in these cases. While 2 of the patients underwent a radical hysterectomy with bilateral salpingo-oophorectomy plus bilateral pelvic lymphadenectomy, 1 patient underwent a radical hysterectomy with bilateral pelvic lymphadenectomy and the remaining patient received a simple total hysterectomy plus post-operative radiotherapy. Of these patients, only 1 had been correctly diagnosed pre-operatively. In 2 patients, the biopsy results had been interpreted as cervical adenocarcinoma, and in the third, the biopsy result was of cervical intraepithelial neoplasia. All 4 patients presented with cervical wall invasion, including invasion of the inner two-thirds in 1 patient. No lymphovascular space invasion or lymph node metastasis was detected. The follow-up time ranged from 49 to 83 months (median, 64 months), and the patients are currently alive and well, with no evidence of recurrent disease. Taking these results as a whole, VGPA is an uncommon type of cervical adenocarcinoma, characterized by its excellent prognosis. HPV infection is associated with the molecular pathogenesis of VGPA, while oral contraceptive use can be excluded. As the disease has a low pre-operative diagnostic accuracy, frequent cervical wall invasion and concomitant lesions, conservative treatment strategies should be carefully considered.
\end{abstract}

Correspondence to: Professor Xue-Qing Wu, Department of Obstetrics and Gynecology, The First Affiliated Hospital of Wenzhou Medical University, 2 Fu-Xue Street, Lu-Chen, Wenzhou, Zhejiang 325000, P.R. China

E-mail: wuxueqing.37@hotmail.com

Key words: diagnosis, uterine cervix, villoglandular papillary adenocarcinoma

\section{Introduction}

Villoglandular papillary adenocarcinoma (VGPA) is a rare but well-differentiated subtype of cervical adenocarcinoma, which accounts for 3.7-4.8\% of cervical adenocarcinomas (1). The disease was described for the first time by Young and Scully (2) in 1989, and predominantly affects young women (2-7). Based on its infrequent lymphovascular space invasion and lymph node metastasis, VGPA is generally considered to have relatively indolent behavior and an excellent prognosis compared with other types of cervical adenocarcinomas (2-4,8-10). The conservative management of VGPA is widely recognized due to its favorable outcome and young patient age, particularly in those individuals who want to preserve their fertility $(2,3,11)$. Nevertheless, risk factors of a poor outcome, including lymphovascular space invasion, lymph node metastasis, tumor recurrence and even mortality, have been reported in several cases (4-8,12-16). Etiologically, the association between HPV infection and VGPA has been demonstrated by certain studies $(1,17,18)$. Controversy remains over whether oral contraceptive use plays a role in the progress of VGPA $(1,3,6)$.

Histologically, VGPA is characterized by a papillary exophytic architecture lined by stratified epithelial cells with mild to moderate nuclear atypia (2). In spite of this, pre-operative diagnosis is usually based solely on a small punch biopsy, which makes it a challenge for gynecologists to promote diagnostic accuracy. In the present study, 4 new cases of VGPA were retrospectively reviewed from inpatient files of The First Affiliated Hospital of Wenzhou Medical University (Wenzhou, Zhejiang, China) from between 2001 and 2012. Written informed consent was obtained from all patients. The aim of this study was to investigate the clinicopathological features, management and prognosis of VGPA of the uterine cervix. In addition, the literature of this infrequent tumor is reviewed.

\section{Case report}

Case 1. A 50-year-old female (gravida 2, para 2) presented to the Gynecologic Clinic of The First Affiliated Hospital of Wenzhou Medical University on July 18, 2007, with a history of postcoital bleeding. The patient had no history of oral contraceptive use. Clinical examination revealed a $1.5 \times 1.5-\mathrm{cm}$ cervical papillary neoplasm and a free parametrium. Cervical 
biopsy showed cervical severe chronic inflammation, with low-grade cervical intraepithelial glandular neoplasia, focal areas of moderate-severe dysplasia of the glandular epithelium and small focal areas of cervical intraepithelial neoplasia (CIN) stage I-II. The patient was initially diagnosed with CIN and subsequently underwent a simple total hysterectomy. The surgical specimen revealed VGPA of the uterine cervix, characterized by finger-like papillae with mild to moderate nuclear atypia, invading the inner two-thirds of the cervical wall, together with CIN stage I-II. Neither lymphovascular space invasion nor lymph node metastasis was detected. In situ hybridization showed HPV infection was negative. The patient received post-operative external radiation treatment (details unknown) due to deep invasion of the cervical wall. The patient shows no evidence of tumor recurrence or metastasis at 74 months post-surgery.

Case 2. A 52-year-old female (gravida 4, para 3) came to our attention on May 29,2009, due to indications of atypical squamous cells on a routine cervical Thinprep cytological test. Prior to the Thinprep cytological test, the patient once received a subtotal hysterectomy due to the fibromyoma of uterine in The First Affiliated Hospital of Wenzhou Medical University. The patient had no history of oral contraceptive use. The HPV test showed a positive result for HPV-16. Gynecological examination showed a $3 \times 3-\mathrm{cm}$ cervical cauliflower-like neoplasm with a free parametrium. A colposcopy with multiple biopsies was performed. The pathological result revealed a type of well-differentiated papillary endocervical adenocarcinoma, with chronic inflammation of the cervical mucosa. Therefore, the initial diagnosis was of a cervical adenocarcinoma. The patient underwent a radical hysterectomy with bilateral salpingo-oophorectomy and bilateral pelvic lymphadenectomy. The surgical specimen revealed a neoplasm with papillary structures superficially invading the surface of the cervical wall. The papillae were thin and tall, and nuclear atypia was mild to moderate. No lymphovascular space invasion or lymph node metastasis was detected. Thus, the specimen was diagnosed as VGPA. The patient remains alive with no evidence of recurrent disease after a 51-month follow-up period.

Case 3. A 70-year-old female (gravida 6, para 6) was referred to The First Affiliated Hospital of Wenzhou Medical University on September 26, 2006, due to abnormal vaginal discharge that had persisted for 6 months, with a bulge in the lower abdomen. The discharge was a moderate amount, and was white and thin. The patient had no history of oral contraceptive use. Gynecological examination showed a $1.5 \times 1.0-\mathrm{cm}$ cervical papillary neoplasm with a free parametrium. The patient underwent a biopsy examination, which resulted in an initial diagnosis of cervical papillary adenocarcinoma. A radical hysterectomy with bilateral salpingo-oophorectomy and bilateral pelvic lymphadenectomy was performed. Intraoperative frozen section revealed a VGPA of the uterine cervix. Post-surgery, the final pathological examination showed a VGPA of the uterine cervix, with superficial invasion of the cervical wall. The VGPA was diagnosed by the presence of thin and tall papillae lined by stratified columnar cells with mild nuclear atypia. No lymphovascular space invasion or

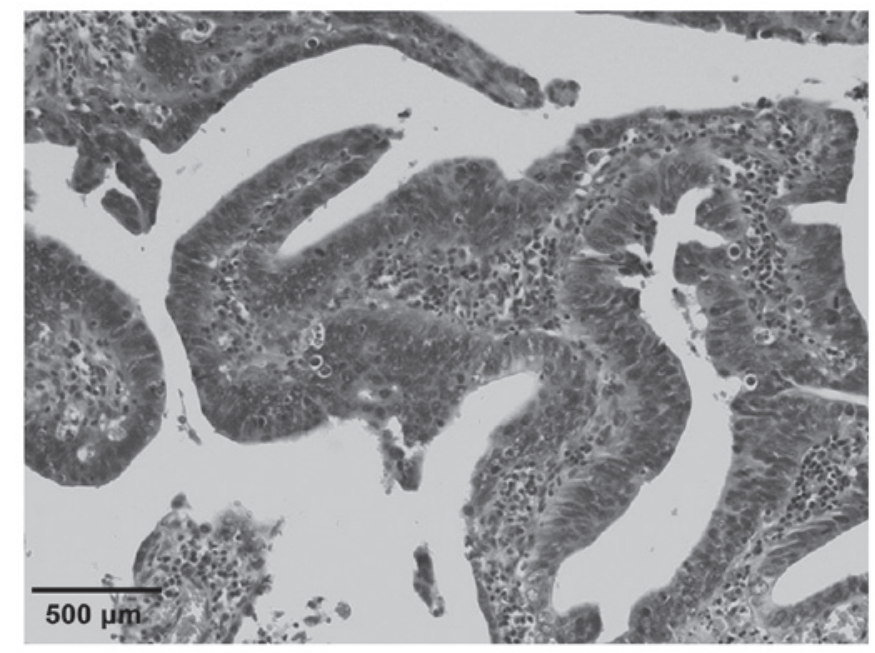

Figure 1. Villoglandular papillary adenocarcinoma with thin and tall papillae. The cells showed mild nuclear atypia (magnification, x100).

lymph node metastasis was detected. HPV types 16, 18, 31 and 33 were strongly positive, as detected by in situ hybridization. The patient currently shows no evidence of tumor recurrence or metastasis at 83 months post-surgery.

Case 4. A 47-year-old female (gravida 2, para 2) presented to the Gynecological Clinic of The First Affiliated Hospital of Wenzhou Medical University on November 25, 2009, with a history of postcoital bleeding. The patient had no history of oral contraceptive use. Gynecological examination revealed a $2.5 \times 2.0-\mathrm{cm}$ cervical neoplasm and a free parametrium. A colposcopy with multiple biopsies was performed. The pathological examination revealed a villoglandular architecture with mild to moderate cellular atypia, indicating a VGPA of the uterine cervix. The HPV test was positive for types 16, 56 and 66. The patient received a radical hysterectomy with bilateral pelvic lymphadenectomy. The surgical specimen was consistent with a VGPA, with superficial invasion of the cervical wall. Neither lymphovascular space invasion nor lymph node metastasis was detected. At 49 months post-surgery, the patient remains alive with no evidence of disease.

Clinicopathological findings. The details of the clinical findings, pathological features and management of the disease are listed in Table I. The median age of the patients was 55 years, with a range of 47-70 years, and all 4 tumors were stage $\mathrm{Ib}$ disease. HPV infection, mainly type 16 , was detected in 3 patients, and 2 patients presented with a mixed HPV infection. While 1 patient was infected with HPV types 16,18 , 31 and 33, the other was infected with HPV types 16,56 and 66. No history of oral contraceptive use was found in these cases. Radical hysterectomy with bilateral salpingo-oophorectomy plus bilateral pelvic lymphadenectomy was used in 2 patients, 1 patient underwent a radical hysterectomy with bilateral pelvic lymphadenectomy, and the remaining patient received a simple total hysterectomy plus post-operative radiotherapy. Of these cases, only 1 patient had been correctly diagnosed pre-operatively. In 2 patients, the biopsy results had been interpreted as cervical adenocarcinoma, and in the third patient, the biopsy result was of CIN. The patients are currently alive with 
no evidence of recurrent disease. The follow-up time ranged between 49 and 83 months (median, 64 months).

On gross examination, the tumors of the VGPA were mainly the exophytic type. The maximum diameter of all tumors ranged between 1.5 and $3 \mathrm{~cm}$ (mean, $2.1 \mathrm{~cm}$ ). Microscopically, VGPA showed thin and tall papillae lined by stratified columnar cells that were of the endocervical type, with mild nuclear atypia (Fig. 1). While 3 tumors were superficially invasive, 1 invaded the inner two-thirds of the cervical wall. Only 1 tumor had an additional lesion (CIN). None of these patients were positive for lymphovascular space invasion or lymph node metastasis.

\section{Discussion}

VGPA of the uterine cervix is extremely rare. Since it was first described by Young and Scully (2) in 1989, few case series have been reported worldwide. Clinically, VGPA is most prevalent in younger women compared with common types of cervical adenocarcinoma (2-7). Young and Scully (2) described 13 cases, with a mean age of 33 years. Lataifeh et al (7) reported a mean age of 38 years in a study of 28 cases. However, the mean patient age in the present study was 55 years, with a range of 47-70 years, which is the maximum average age to date. The clinical manifestations of VGPA are non-specific, and the majority of cases show abnormal vaginal bleeding, postcoital bleeding or abnormal vaginal discharge.

Controversy remains over whether VGPA could be correlated to oral contraceptive use. Brinton et al (19) demonstrated that oral contraceptive use was a major risk for adenocarcinoma, while Jones et al (3) reported that 15 out of 24 patients with VGPA had a history of oral contraceptive use. However, Young and Scully (2) reported that only 1 out of 13 patients with VGPA had a history of oral contraceptive use. Giordano et al (1) and Hagiwara et al (6) reported that no patient had taken oral contraceptives prior to diagnosis. In the present cases, all 4 patients denied a history of oral contraceptive use. Therefore, the effect of oral contraceptive use on the development of VGPA remains suspect. Further studies are required to confirm this association in VGPA cases.

HPV is well recognized and its role in cervical carcinogenesis has been proven (20). To the best of our knowledge, only 3 studies have referred to the association between VGPA and HPV infection $(1,17,18)$. Jones et al $(17)$ retrospectively analyzed 12 cases of VGPA for presence of HPV. HPV was present in all tumors and consisted of type $16(42 \%)$ and type $18(58 \%)$ strains. Yamazawa et al (18) reported a case of VGPA that was positive for HPV type 18. Giordano et al (1) revealed a strong positive signal for HPV-DNA, using polymerase chain reaction (PCR) amplification of tumoral VGPA DNA. In the present study, 3 patients presented with HPV infection, mainly type 16 , and 2 of these presented with a mixed type. Considering these positive results in the present patients, and in accordance with previous studies, it is reasonable to support HPV infection, particularly high-risk types, in the molecular pathogenesis of this unusual cervical adenocarcinoma.

Compared with the common types of cervical adenocarcinomas, VGPA is morphologically featured by a predominant villoglandular papillary growth pattern $(2,3,8)$. Upon gross 
examination, the tumors are mostly polypoid or papillary neoplasms, but occasionally are infiltrative and friable lesions. Microscopically, VGPA shows a villoglandular pattern lined by stratified epithelial cells, with mild to moderate nuclear atypia. The majority of VGPA cases present with only superficial invasion of the cervical wall. In the case series of Young and Scully (2) and Jones et al (3), 9 tumors with no stromal invasion, 24 tumors with superficial invasion confined to the inner third of the cervical wall and 3 tumors with deep invasion of the cervical wall were described. In the present study, 3 tumors showed superficial invasion and 1 tumor expressed invasion in the inner two-thirds of the cervical wall. VGPA has been reported to be associated with other lesions. Heatley (10) retrospectively collected 140 cases of VGPA and found that 40 cases $(28.6 \%)$ were associated with squamous or glandular intraepithelial neoplasia. Moreover, to date, various lesions have been described in at least 12 out of 173 cases, including squamous carcinoma, glandular carcinoma, adenosquamous carcinoma and small cell carcinoma $(14,21-24)$. In the present study, the pathological analysis of the cervical biopsy showed VGPA associated with CIN in case 1.

Young and Scully (2) stated that the term VGPA should be reserved for those tumors in which the villoglandular component was the exclusive or almost exclusive pattern; this has additionally been supported by other studies $(3,8)$. In spite of this, the low diagnostic accuracy means the diagnosis of VGPA is a big challenge. Korach et al (5) reviewed the cases of 9 patients in whom the final pathological results confirmed VGPA. Of these, the correct pre-operative diagnosis had only bee made in 2 cases, while the initial biopsy results were benign or premalignant in three further cases. The remaining 4 patients were initially misdiagnosed with other malignant tumors. Alfsen et al (25) reevaluated the classification of non-squamous cell carcinomas of the uterine cervix. Only 3 out of 15 cases with VGPA were agreed upon by reviewers. In the present study, only 1 out of 4 patients was correctly diagnosed pre-operatively. This challenge was mainly due to the limited biopsy specimens making it hard to exclude invasive lesions. Additionally, as the disease has a low incidence rate, pathologists may not regard VGPA as a distinct entity. Thus, the present study emphasizes the importance of an awareness of this rare type of cervical carcinoma. Due to the high frequency of cervical wall invasion and the coexistence of other neoplastic components, as aforementioned, the pre-operative diagnosis should not be based only on a small punch biopsy and a cervical conization is strongly recommended.

Generally, the outcome of cervical adenocarcinoma is considered to be worse than that of squamous cell carcinoma (26). However, VGPA has been reported to have a limited metastatic potential and an excellent prognosis $(2-4,8-10)$. Jones et al (17) verified the absence of an associated oncogene (c-erbB-2 and int-2) or tumor suppressor gene (p53, MCC, APC and BRCA1) in the tissues of VGPA by PCR amplification, supporting the indolent behavior and the favorable outcome of this unusual tumor. More conservative treatment has also been performed, particularly in patients who want to preserve their fertility $(2,3,11)$. Nevertheless, 12 cases with lymph node metastasis and 13 cases with recurrence have been reported in the English literature $(4-8,12,16)$, evoking caution with regard to conducting less radical surgery options such as conization. Lai et al (4) suggested the use of a radical hysterectomy plus salpingo-oophorectomy with bilateral pelvic lymphadenectomy in the management of early-stage VGPA, which resulted in a 5-year survival time in the majority of patients. All the patients in the present study underwent a radical procedure, except one. The long-term follow-up (median, 64 months) supported a favorable outcome of VGPA.

In summary, the present study describes the clinicopathological features of 4 new cases of VGPA, and indicates an association between HPV infection and VGPA, but not between oral contraceptive use and VGPA. Despite its excellent prognosis, VGPA presents with frequent cervical wall invasion and concomitant lesions, meaning that care must be taken prior to choosing any conservative therapy. VGPA is a rare and distinct type of cervical carcinoma. A greater awareness and more surgical specimens will benefit its diagnosis.

\section{Acknowledgements}

This study was sponsored by grants from the National Natural Science Foundation of China (no. 81100392), the Zhejiang Provincial Natural Science Foundation of China (no. Y2110606), the China Higher Specialized Research Fund of the Doctoral Program (no. 20113321120004) and the Returned Overseas Chinese Ministry of Education Scientific Research Foundation (no. 2012-1707).

\section{References}

1. Giordano G, D'Adda T, Gnetti L, Merisio C, Gabrielli M and Melpignano M: Villoglandular adenocarcinoma of the cervix: Two new cases with morphological and molecular study. Int J Gynecol Pathol 26: 199-204, 2007.

2. Young RH and Scully RE: Villoglandular papillary adenocarcinoma of the uterine cervix. A clinicopathologic analysis of 13 cases. Cancer 63: 1773-1779, 1989.

3. Jones MW, Silverberg SG and Kurman RJ: Well-differentiated villoglandular adenocarcinoma of the uterine cervix: A clinicopathological study of 24 cases. Int J Gynecol Pathol 12: 1-7, 1993.

4. Lai JCY, Chen JR, Chen YJ, Hsu CH, Wang TY, Yang YC, Su TH, Tsai TH and Wang KL: Villoglandular Adenocarcinoma of the uterine cervix: An analysis of 12 clinical cases. Int J Gerontol 5: 49-52, 2011.

5. Korach J, Machtinger R, Perri T, Vicus D, Segal J, Fridman E and Ben-Baruch G: Villoglandular papillary adenocarcinoma of the uterine cervix: A diagnostic challenge. Acta Obstet Gynecol Scand 88: 355-358, 2009.

6. Hagiwara T, Kaku T, Kobayashi H, Wake N and Saito T: Well-differentiated villoglandular adenocarcinoma of the uterine cervix: Assessment of cytological features by histological subtypes. Acta Cytol 57: 61-68, 2013.

7. Lataifeh IM, Al-Hussaini M, Uzan C, Jaradat I, Duvillard P and Morice P: Villoglandular papillary adenocarcinoma of the cervix: A series of 28 cases including two with lymph node metastasis. Int J Gynecol Cancer 23: 900-905, 2013.

8. Utsugi K, Shimizu Y, Akiyama F, Umezawa S and Hasumi K: Clinicopathologic features of villoglandular papillary adenocarcinoma of the uterine cervix. Gynecol Oncol 92: 64-70, 2004.

9. Khunamornpong S, Siriaunkgul S and Suprasert P: Well-differentiated villoglandular adenocarcinoma of the uterine cervix: Cytomorphologic observation of five cases. Diagn Cytopathol 26: 10-14, 2002.

10. Heatley MK: Villoglandular adenocarcinoma of the uterine cervix-a systematic review of the literature. Histopathology 51: 268-269, 2007.

11. Falcón O, García R, Lubrano A, Morín JC and Andujar M: Successful term delivery following conservative management for villoglandular papillary adenocarcinoma of the uterine cervix: A case report. Gynecol Oncol 101: 168-171, 2006. 
12. Dede M, Deveci G, Deveci MS, Yenen MC, Goktolga U, Dilek S and Gunhan O: Villoglandular papillary adenocarcinoma of the uterine cervix in a pregnant woman: A case report and review of literature. Tohoku J Exp Med 202: 305-310, 2004.

13. Heron DE, Axtel A, Gerszten K, Amortegui A, Kelley J, Comerci J and Edwards RP: Villoglandular adenocarcinoma of the cervix recurrent in an episiotomy scar: A case report in a 32-year-old female. Int J Gynecol Cancer 15: 366-371, 2005.

14. Macdonald RD, Kirwan J, Hayat K, Herrington CS and Shawki H: Villoglandular adenocarcinoma of the cervix:clarity is needed on the histological definition for this difficult diagnosis. Gynecol Oncol 100: 192-194, 2006.

15. Rubesa-Mihaljević R, Vrdoljak-Mozetic D, Ostojić DV, Stemberger-Papić S, Sindik N and Krasević M: Villoglandular papillary adenocarcinoma of the uterine cervix with aggressive clinical course-a case report. Coll Antropol 34: 291-294, 2010.

16. Takai N, Hayashita C, Nakamura S, Narahara H and Matsumoto H: Villoglandular papillary adenocarcinoma of the uterine cervix diagnosed during pregnancy. Eur J Gynaecol Oncol 31: 573-574, 2010.

17. Jones MW, Kounelis S, Papadaki H, Bakker A, Swalsky PA, Woods J and Finkelstein SD: Well-differentiated villoglandular adenocarcinoma of the uterine cervix: Oncogene/tumor suppressor gene alterations and human papillomavirus genotyping. Int J Gynecol Pathol 19: 110-117, 2000.

18. Yamazawa K, Matsui H, Seki K, Mitsuhashi A, Kawamata Y, Shirasawa $\mathrm{H}$ and Sekiya S: Human papillomavirus-positive well-differentiated villoglandular adenocarcinoma of the uterine cervix: A case report and review of the literature. Gynecol Oncol 77: 473-477, 2000
19. Brinton LA, Herrero R, Reeves WC, de Britton RC, Gaitan E and Tenorio F: Risk factors for cervical cancer by histology. Gynecol Oncol 51: 301-306, 1993.

20. Kleter B, van Doorn LJ, ter Schegget J, Schrauwen L, van Krimpen K, Burger M, ter Harmsel B and Quint W: Novel short-fragment PCR assay for highly sensitive broad-spectrum detection of anogenital human papillomaviruses. Am J Pathol 153: 1731-1739, 1998.

21. Fadare $\mathrm{O}$ and Zheng W: Well-differentiated papillary villoglandular adenocarcinoma of the uterine cervix with a focal high-grade component: Is there a need for reassessment? Virchows Arch 447: 883-887, 2005.

22. Bouman A, Oosterhuis GJ, Naudin ten Cate L and van Doorn GA: Villoglandular papillary adenocarcinoma of the cervix. Beware of a wolf in sheep's clothing. Eur J Obstet Gynecol Reprod Biol 87: 183-189, 1999.

23. Collinet P, Prolongeau JF and Vaneecloo S: Villoglandular papillary adenocarcinoma of the uterine cervix. Eur J Obstet Gynecol Reprod Biol 86: 101-103, 1999.

24. Kaku T, Kamura T, Shigematsu T, Sakai K, Nakanami N, Uehira K, Amada S, Kobayashi H, Saito T and Nakano H: Adenocarcinoma of the uterine cervix with predominantly villoglandular papillary growth pattern. Gynecol Oncol 64: 147-152, 1997.

25. Alfsen GC, Reed W and Abeler VM: Reproducibility of classification in non-squamous cell carcinomas of the uterine cervix. Gynecol Oncol 90: 282-289, 2003.

26. Costa MJ, McIlnay KR and Trelford J: Cervical carcinoma with glandular differentiation: histological evaluation predicts disease recurrence in clinical stage I or II patients. Hum Pathol 26: 829-837, 1995. 